\title{
Butterfly in the winds
}

Nature 556, 345-348 (2018)

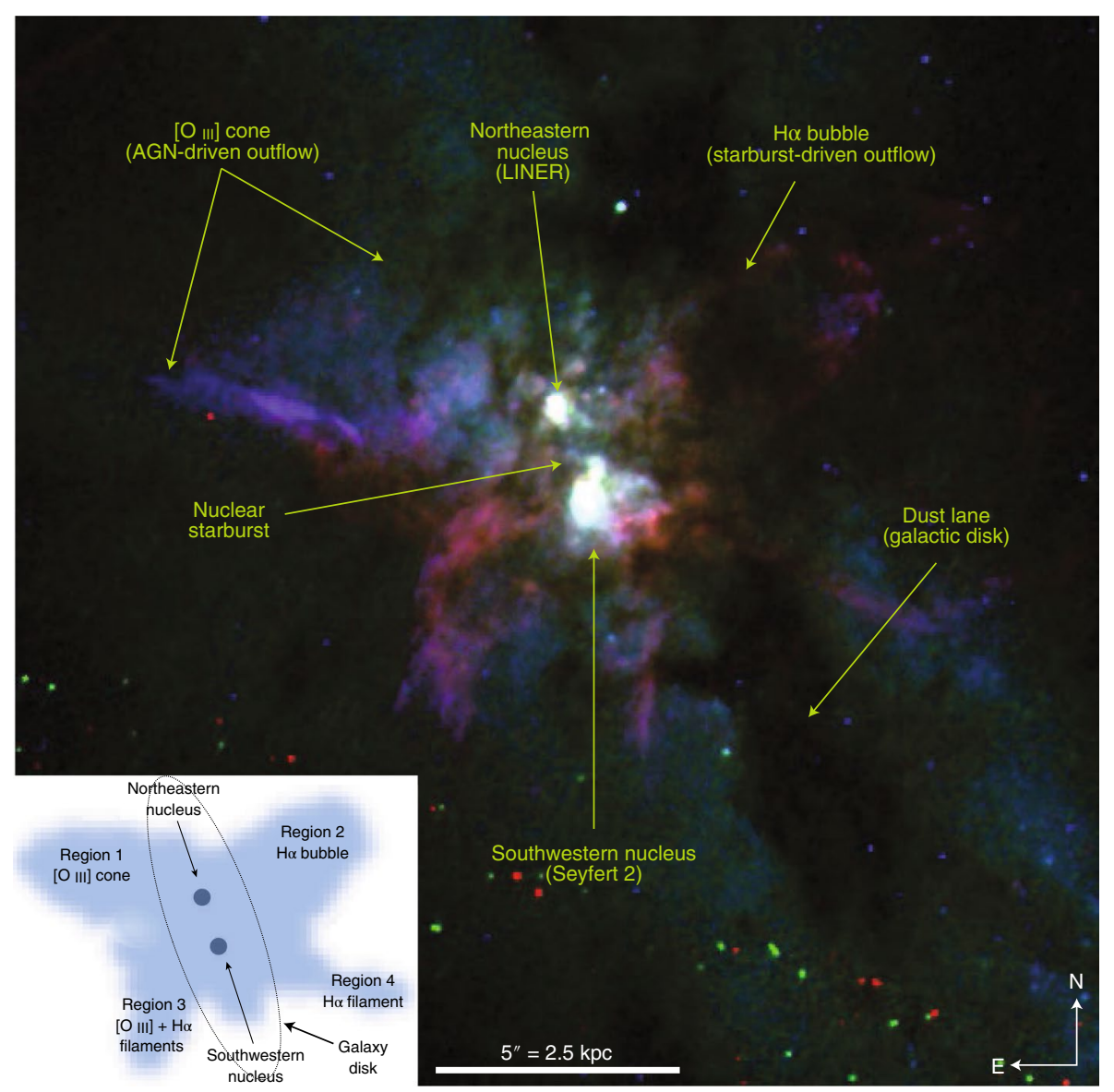

Credit: Macmillan Publishers Ltd

Mergers of gas-rich galaxies have long been considered as a key stage in galactic evolution - they funnel copious amounts of gas toward the centre of the merging system facilitating both intense star formation and the (potentially synchronous) triggering of an active galactic nucleus (AGN). Francisco Müller-Sánchez and colleagues performed narrow-band photometric and spectroscopic observations of the archetypical merging system NGC 6240 and revealed the emergence of two gas outflows: one driven by the actively accreting supermassive black holes and the other powered by the ongoing starburst.

NGC 6240 hosts a binary supermassive black hole and shows signs of ongoing star formation with rates exceeding one hundred solar masses per year. Hubble Space Telescope observations revealed a complex, 'butterfly-like' morphology (pictured) for the narrow-line emitting gas surrounding the binary AGN. The authors used photoionization modelling to infer that the northeastern 'wing' of the butterfly is ionized by the AGNs, whereas the southeastern and southwestern wings appear to be heated by star-formation-driven shocks.

The derived gas kinematics revealed clear signatures of outflows. With projected velocities of up to $1,200 \mathrm{~km}$ per second and a mass outflow rate of up to 75 solar masses per year, the AGN-driven outflow to the northeast dominates the energy budget of the system. Considered together with the starburst-driven outflow (itself carrying away roughly 30 solar masses per year), their combined effect was found to remove enough material to curb the growth of the merging system. As such, the authors present a snapshot of the long-sought phase of star-formation suppression in gasrich galactic mergers.

Marios Karouzos

Published online: 30 April 2018

https://doi.org/10.1038/s41550-018-0480-6 